\title{
Une ou des politiques publiques nationales de l'ESS ?
}

Trente ans de tâtonnements politiques et administratifs

\section{A national policy or a patchwork of measures on the social and solidarity economy? Thirty years of political and administrative trial and error}

\section{Scarlett Wilson-Courvoisier}

Numéro 325, juillet 2012

ESS : de l'approche entrepreneuriale à une perspective institutionnaliste

The social and solidarity economy: From the entrepreneurial approach to an instutitionalist perspective

URI : https://id.erudit.org/iderudit/1017422ar

DOI : https://doi.org/10.7202/1017422ar

Aller au sommaire du numéro

Éditeur(s)

Association Recma

ISSN

1626-1682 (imprimé)

2261-2599 (numérique)

Découvrir la revue

Citer cet article

Wilson-Courvoisier, S. (2012). Une ou des politiques publiques nationales de l'ESS ? Trente ans de tâtonnements politiques et administratifs. Revue internationale de l'économie sociale, (325), 78-93.

https://doi.org/10.7202/1017422ar
Résumé de l'article

Il n'existe pas aujourd'hui en France une politique publique de l'économie sociale et solidaire (ESS). Pour qu'il y ait politique publique, il faut qu'il y ait reconnaissance de la nécessité à en créer une et volonté politique pour la mener, sans omettre de la doter des moyens nécessaires et suffisants pour qu'elle soit efficace et qu'elle s'impose à tous. Ces conditions sont-elles réunies en France ? La question suppose de revenir dans un premier temps sur l'histoire récente des relations institutionnelles entre l'ESS et les pouvoirs publics : les difficultés à positionner l'objet de l'ESS dans les dispositifs publics, de la naissance de la Délégation interministérielle à l'économie sociale (Dies) au rapport Vercamer ; puis sur la variabilité des représentations sous-jacentes en ce qui concerne le périmètre de l'ESS et son rattachement administratif, à travers les divers lois et décrets relatifs au secteur. De cet examen, il ressort que, depuis 1991, l'économie sociale n'a jamais été considérée dans l'ensemble de ses dimensions. Son morcellement explique sans doute en partie la transformation sur trente ans des modes de relation entre l'ESS et la puissance publique, de la cogestion à la mise en concurrence. 


\section{UNE OU DES POLITIQUES PUBLIOUES NATIONALES DE L'ESS?}

Trente ans de tâtonnements politiques et administratifs

*Ex-conseillère technique à la Délégation interministérielle à l'économie sociale (Dies). Mél.: Scarlett.wilson@orange.fr. par Scarlett Wilson-Courvoisier*

Il n'existe pas aujourd'hui en France une politique publique de l'économie sociale et solidaire (ESS). Pour qu'il y ait politique publique, il faut qu'il y ait reconnaissance de la nécessité à en créer une et volonté politique pour la mener, sans omettre de la doter des moyens nécessaires et suffisants pour qu'elle soit efficace et qu'elle s'impose à tous. Ces conditions sont-elles réunies en France? La question suppose de revenir dans un premier temps sur l'bistoire récente des relations institutionnelles entre l'ESS et les pouvoirs publics: les difficultés à positionner l'objet de l'ESS dans les dispositifs publics, de la naissance de la Délégation interministérielle à l'économie sociale (Dies) au rapport Vercamer; puis sur la variabilité des représentations sous-jacentes en ce qui concerne le périmètre de l'ESS et son rattachement administratif, à travers les divers lois et décrets relatifs au secteur. De cet examen, il ressort que, depuis 1991, l'économie sociale n'a jamais été considérée dans l'ensemble de ses dimensions. Son morcellement explique sans doute en partie la transformation sur trente ans des modes de relation entre l'ESS et la puissance publique, de la cogestion à la mise en concurrence.

I I n'existe pas aujourd'hui en France une politique publique de l'économie sociale et solidaire (ESS). Il existerait plutôt des politiques publiques - encore que cette affirmation même mériterait débat - qui concernent certains secteurs et acteurs de l'ESS. En 1981, avait été imaginée et lancée une politique publique globale qui, rencontrant très rapidement des limites, s'est essoufflée.

Dès 1983-1984, en effet, la donne n'était plus la même. A deux reprises, une nouvelle dynamique de politique publique fut retrouvée, mais sur la base de nouvelles orientations et dans un contexte économique et social profondément modifié.

Au cours de la période 1998-2001, durant laquelle Hugues Sibille fut en charge de la Délégation interministérielle à l'innovation, à l'expérimentation sociale et à l'économie sociale (Diieses) au sein du gouvernement de Lionel Jospin, l'accent fut mis sur le seul secteur associatif.

Ensuite, durant la période 2001-2003, l'économie solidaire fut considérée comme prioritaire avec la création d'un secrétariat d'Etat, confié à Guy Hascoët, toujours au sein du gouvernement Jospin. 
Enfin, la période actuelle est riche de contradictions et de paradoxes, intéressants pour des recherches, mais peut-être trop nombreux pour faciliter l'imagination d'une véritable politique publique structurante. C'est en effet au moment où la "crise " suscite un regain d'intérêt pour une ESS devenue aussitôt « tendance » que l'ESS se retrouve interpellée cette fois-ci par le mouvement des entrepreneurs sociaux et les travaux du Labo de l'ESS de Claude Alphandéry, tandis que les représentants des mouvements coopératifs ne siègent plus au sein du Conseil des entreprises, employeurs et groupements de l'économie sociale (Ceges), construit en 2002 pour représenter l'ESS dans son ensemble.

Pour qu'il y ait politique publique, un certain nombre de conditions doivent être réunies, et les partenaires clairement identifiés et impliqués. En résumé, il faut qu'il y ait reconnaissance d'une nécessité à créer et à mener une politique publique dans tel ou tel secteur d'activité et volonté politique pour la conduire, sans omettre de la doter des moyens nécessaires et suffisants pour qu'elle soit efficace et qu'elle s'impose à tous. Selon Rafael H. Chavez (2011), quatre phases seraient nécessaires, mais le passage de l'une à la suivante est loin d'être évident. Il faut d'abord une sensibilisation des élus, ensuite une inclusion de l'ESS dans les politiques publiques et l'implication des élus, puis une construction de ces politiques publiques et, enfin, des budgets suffisants pour pouvoir les mener. Par ailleurs, selon lui, l'existence de personnes «moteurs-catalyseurs " est essentielle.

Ces conditions sont-elles réunies en France? La question suppose de revenir sur l'histoire récente des relations institutionnelles entre l'ESS et les pouvoirs publics: sur les difficultés à positionner l'objet de l'ESS dans les dispositifs publics, de la naissance de la Délégation à l'économie sociale au rapport Vercamer (première partie); sur la variabilité des représentations sous-jacentes en ce qui concerne son périmètre et son rattachement administratif, à travers les divers lois et décrets relatifs au secteur (deuxième partie). De cet examen, il ressort que, depuis 1991, l'économie sociale n’a jamais été considérée dans l'ensemble de ses dimensions. Son morcellement explique sans doute en partie la transformation sur trente ans des modes de relation entre l'ESS et la puissance publique, de la cogestion à la mise en concurrence (troisième partie).

\section{De la redécouverte de l'économie sociale au rapport Vercamer}

La décennie 70 est marquée par la redécouverte du thème gidien d'économie sociale (Pénin, 2006). La volonté de certains représentants de groupements nationaux (de la génération des leaders politiques charismatiques) permet la redécouverte d'un passé commun, autour d'un "étendard conceptuel " redéfini avec l'aide d'intellectuels tels Henri Desroche, Claude Vienney, Lucien Pfeiffer... (Chomel, 1994; Hipszman, 2003). A partir de la rencontre de quelques hommes 
(1) Avec d'autres administrations de mission: la Délégation interministérielle à la ville (DIV), la Délégation interministérielle à l'aménagement du territoire et à l'attractivité régionale (Datar), devenue temporairement Délégation interministérielle à l'aménagement et à la compétitivité des territoires (Diact). politiques sensibles à ce concept, ils préparent ensemble une politique publique qui passe par la reconnaissance officielle de l'économie sociale (ES). Mais la reconnaissance de son objet principal ne cessera de varier de 1981 à 2010 (de la démocratie économique à l'économie de la solidarité et à la cohésion sociale), du fait des mutations idéologiques, sociologiques et économiques de ces trente dernières années.

\section{De la démocratie économique...}

En 1981, l'ES figure à la soixante-deuxième place des cent dix propositions du candidat François Mitterrand, héritées du programme commun abandonné en 1977, au chapitre "La démocratie économique, des droits nouveaux pour les travailleurs ": "La gestion du secteur public sera largement décentralisée. Les instances de direction des entreprises seront soit tripartites (collectivités publiques, travailleurs, usagers), soit formées par la coexistence d'un conseil de gestion élu par les travailleurs et d'un conseil de surveillance. Les représentants des travailleurs seront élus directement à la proportionnelle et à la plus forte moyenne. Des conseils d'unité et d'atelier élus par les travailleurs seront instaurés. Un secteur d'économie sociale fondé sur la coopération et la mutualité expérimentera des formes nouvelles d'organisation des travailleurs. " Le Premier ministre, Pierre Mauroy, et le secrétaire d'Etat au Plan et à l'Aménagement du territoire, Michel Rocard (2001), chargé de l'Economie sociale, mettent en œuvre une politique en créant un outil qui est une administration de mission et non de gestion ${ }^{(1)}$, avec peu de personnel (trois personnes) et un budget minimaliste qui ne permet pas d'engager de grandes actions. Il s'agit de convaincre, d'impulser, de coordonner, de trouver les effets leviers et, par le dialogue interministériel, de réduire les freins au développement de l'ES. Il s'agit aussi d'aider les acteurs à se fédérer. Cette politique est très centrée sur le développement économique des trois familles (les coopératives, les mutuelles et les associations), comme on disait à l'époque, et marquée par le contexte historique récent: l'autogestion, les débats liés à l'économie administrée par l'Etat, un puissant secteur public (nationalisation), et le capitalisme. On parle d'ailleurs également de tiers secteur (Jacques Delors).

L'action va donc logiquement concerner en priorité un plan de développement des Scop avec soutien financier, suivi d'un plan de formation du mouvement et d'un travail législatif intense, qui aboutira notamment à la loi du 20 juillet 1983 sur la modernisation des coopératives d'entreprises et première loi d'économie sociale (Soulage, Hipszman, 2004), ainsi qu'à la création de l'outil Ides (Institut de développement de l'économie sociale, www.esfin-ides.com), en 1984, et du titre participatif par une loi, également de 1983 (Hiez, 2005). Lorigine est clairement identifiable au niveau national, que ce soit du côté des acteurs rassemblés au sein du Comité national de liaison des activités mutualistes, coopératives et associatives (Cnlamca) ou du côté de l'Etat, qui prend des dispositions pour jeter les bases d'une future politique territoriale 
(2) Dans les années 70, des groupements régionaux de la coopération maillaient le territoire national. Ouverts au mouvement mutualiste (GRCM), puis au mouvement associatif (GRCMA), ils deviennent les chambres régionales de l'économie sociale (Cres) en 1993 (NDLR).
(3) Le 8octobre 2008, I'Association des régions de France publie un manifeste pour une économie sociale et solidaire; voir " Actualités ", Recma, $n^{\circ} 311$ (NDLR). de développement de l'ES par la création d'un réseau de correspondants régionaux auprès des préfets, afin de mettre en place les premiers axes de cette politique, telle la proposition, dès 1982, de créer avec les groupements régionaux de la coopération, de la mutualité et des associations $\left(\mathrm{GRCMA}^{(2)}\right)$ des agences régionales de développement du secteur. Trop vite, trop tôt.

Cette proposition "avant-gardiste" ne sera mise en œuvre qu'en Lorraine (donnant naissance à la boutique de gestion Alexis, www. alexis.fr). En mai 2010, suite au rapport Vercamer (lire infra), le Conseil national des chambres régionales de l'ESS (CNCres) a annoncé la mise en chantier de vingt-six agences régionales de développement économique de l'économie sociale et solidaire. C'est un beau projet qui, cette fois, pourrait aboutir. Cette politique territoriale de reconnaissance de l'ES et de son développement s'est concrétisée à travers onze contrats de plan Etat-Régions (non poursuivis par la volonté d'un délégué en 2004, mais aussi en raison de mesures de rigueur budgétaire), puis, à partir de 2001, au moyen de conventions pluriannuelles d'objectifs étendues progressivement à l'ensemble des régions (2008), sans compter les appels à projets de l'époque de G. Hascoët, puis de M. Hirsch (lire infra), qui ont insufflé une vraie dynamique territoriale, tandis que les régions, dans leur ensemble, décidaient d'une politique de soutien à l'économie sociale et solidaire dès 2004 (les conseils régionaux sont passés à gauche cette année-là (3)). Cette politique d'intégration territoriale de l'ESS s'est traduite concrètement dans certains chapitres des schémas de développement économique régional.

\section{... à l'économie de la solidarité...}

Jacques Delors, ministre de l'Economie et des Finances de 1981 à 1984, se trouve vite dans l'obligation d'opérer un changement radical de politique économique, après une courte période de relance par la consommation. Laurent Fabius devient Premier ministre en juillet 1984, et cette ligne va perdurer, d'autant que le chômage, que l'on croyait conjoncturel, ne cesse d'augmenter, confirmant son caractère structurel. Larrivée de Jean Gatel à la tête d'un secrétariat d'Etat initialement dévolu au Développement local ne satisfait pas les représentants de l'ES, qui se mobilisent pour faire changer l'intitulé en secrétariat d'Etat à l'Economie sociale. Alain de Romefort, directeur de ce cabinet composé de quarante personnes, fait des appels à projets et renvoie pour la première fois la Dies au rôle d'administration de gestion. Ce sera le cas à chaque fois qu'un secrétariat d'Etat sera creé: en 1988 (Tony Dreyfus), en 2000 (Guy Hascoët, dont le cabinet du secrétariat à l'Economie solidaire est également dirigé par Alain de Romefort) et en 2006 (Martin Hirsch).

Les années 1985 à 1995 (François Soulage, délégué en 1989) ciblent l'Europe, la refonte du Code de la mutualité. Déjà pointe l'importance accordée aux associations et au secteur associatif, dynamique en termes de projets, d'initiatives et de création d'activité et d'emplois. 
(4) L'abaissement de la durée légale du travail à 35 heures, loi emblématique du gouvernement Jospin, impacte naturellement I'ESS, comme le reste de l'économie, sans que ses effets sur le monde associatif, le rôle des administrateurs bénévoles dans les mutuelles, etc., aient fait l'objet d'études approfondies à notre connaissance.

(5) Voir Recma, $\mathrm{n}^{\circ} 282$, "Charte d'engagements réciproques entre l'Etat et les associations re-groupées au sein de la CPCA ", 2001 (Amblard 2001, 2002).
A partir de 1997, de grandes lois sont votées: « Nouveaux services emplois jeunes ", "Lutte contre les exclusions », loi Voynet sur "L'aménagement et le développement durable du territoire" (Koulytchizky, 2006), couverture maladie universelle (CMU), qui utilisent les secteurs de l'ESS (associations pour les emplois-jeunes ou la loi Voynet, les mutuelles pour la CMU.... ${ }^{(4)}$.

Les années 1998-2000 sont marquées par la reconnaissance du secteur associatif dans son ensemble, avec notamment la commémoration du centenaire de la loi de 1901, la Charte d'engagements réciproques entre l'Etat et les associations regroupées au sein de la $\mathrm{CPCA}^{(5)}$, les assises départementales et nationales de la vie associative en février 1999, organisées par la Dies, et, en 2000, les consultations régionales de l'ESS, imaginées et organisées par la Dies, et dont la dynamique et la logique vont être impactées par celles des appels à projets "Dynamiques solidaires » de Guy Hascoët. Ces deux derniers événements seront fort heureusement des succès, même s'ils vont réveiller le "débat des anciens et des modernes", de l'ES institutionnelle, dite "œcuménique ", et de l'économie solidaire revendiquée (Laville, 2007; Draperi, 2009). Alors que la lutte contre les exclusions est affirmée comme un objectif politique, l'économie solidaire, en théorisant l'encastrement de l'activité économique dans le lien social de proximité, l'hybridation des ressources et en relativisant ainsi l'importance des statuts au regard des activités menées, met notamment en lumière l'insertion par l'activité économique, qui devient, sur fond de chômage de masse et de diminution de la sphère d'intervention publique, la grande affaire entraînant confusions et a priori chez les décideurs publics: l'ESS, c'est de l'insertion; les entreprises de l'ES font de l'insertion, donc l'ESS est globalement assistée et vit sur l'argent public (Medef, 2002)!

\section{... et à la lutte contre l'exclusion}

La Délégation interministérielle à l'économie sociale, rebaptisée Délégation interministérielle à l'innovation, à l'expérimentation sociale et à l'économie sociale en 1998 et en 2006, entame un chemin de croix. Elle s'épuise entre gestion de dossiers (appels à projets), tentatives d'initier de nouveaux chantiers spécifiques à son " cœur de cible ", défense de ses budgets et de son périmètre, recherche de la mise en cohérence des diverses orientations gouvernementales. L'outil est mal utilisé, décrié, peu soutenu, de moins en moins visible, stigmatisé comme inutile et inefficace (page $98 \mathrm{du}$ rapport de la commission « Familles, vulnérabilité pauvreté ", présidée par Martin Hirsch, 2005). Devenu haut-commissaire aux Solidarités actives, Martin Hirsch utilisera néanmoins les agents de la Diieses et son budget.

En 2010, le gouvernement Fillon choisit, après plusieurs essais de « mise à la casse ", de " dissoudre ", par fusion-absorption au sein d'une Direction générale de la cohésion sociale nouvellement créée, l'outil qu'était la Diieses, créée en 1981 (même s'il est évident qu'après vingt-neuf ans 
(6) «Rapport sur le développement de I'ESS et de l'entrepreneuriat social ", www.recma.org/ node/1040. il fallait la repenser et la restructurer); il choisit aussi de lancer simultanément une mission confiée au député Francis Vercamer ${ }^{(6)}$, qui vise le développement et la promotion de l'ESS comme de l'entrepreneuriat social. Il existe toutefois, au-delà de ces choix politiques circonstanciés, un véritable désengagement de l'Etat (à tout le moins, une certaine amnésie pour ses propres créations), qu'illustre le fonctionnement de l'administration. Son encadrement supérieur "déconnecté du terrain " est souvent davantage préoccupé par les parcours personnels que par la compréhension d'autres modes managériales, par l'innovation et l'intérêt général. Des méthodes et des critères de gestion "copiés-collés" sur l'entreprise privée, qui se révèlent souvent destructeurs pour l'identité et les spécificités de certains secteurs: au niveau national, le secteur sanitaire et social, l'hôpital, par exemple; au niveau des collectivités locales, les marchés publics qui s’adressent aux associations en les dénaturant...

Pourrait s'ajouter à cette liste un gouvernement qui, après avoir « fait son marché » de manière orientée au sein de l'ESS (la formule Scop, pour la reprise ou la transmission d'entreprise, les services à la personne façon Borloo, le caritatif, le philanthropique et le social du social), a bien du mal à considérer la transversalité et la globalité du secteur. Nonobstant, la Diieses peut se prévaloir de certains succès (encadré 1).

\section{Encadré 1 Les réalisations de la Diieses}

- Le soutien aux Cress et la politique territoriale.

- Le programme Recherche et l'observation statistique.

- L'évaluation.

- L'innovation.

- L'interministérialité de son action.

- La participation active à la mission de Francis Vercamer, à l'esprit de cette mission et à ses premiers résultats (les Conseil supérieur de la coopération et Conseil supérieur de l'ESS de nouveau mis au travail).

Dans l'esprit des pionniers de la Dies et jusqu'à Hugues Sibille, le Comité consultatif de l'ES, ancêtre du conseil supérieur, avait précisément comme mission d'apporter les éléments constitutifs d'une future politique publique de l'ES. De même, la méthodologie utilisée pour les assises départementales de la vie associative et les consultations régionales (dernières tentatives conjointes des responsables de l'ESS et de la Dies) était en partie destinée à rassembler d'abord sur le terrain, mais en lien avec le national (comité de pilotage), les éléments d'une politique publique de l'ES dont le gouvernement s'emparerait pour la mettre en œuvre. 


\section{Un périmètre à géométrie variable}

(7) Le « $i$ »'interministérielle ne figure pas dans l'appellation officielle. Rattachée au Premier ministre, la délégation n'en étant pas moins interministérielle de fait, le "i " s'est imposé dans l'acronyme. Lire «Intitulés de la délégation à travers les décrets ", en annexe.
L'incertitude sur l'objet des politiques publiques renforce l'incertitude sur le périmètre de l'économie sociale, sur son identité, et donc sur son rattachement administratif, ce que nous montre la multiplication des décrets, instituant le champ et les missions de la délégation.

\section{D'une acception statutaire de l'économie sociale...}

Le décret du 15 décembre 1981 portant création d'une Délégation à l'économie sociale $\left(\right.$ Dies $\left.^{(7)}\right)$ auprès du Premier ministre et mise à la disposition du ministre du Plan et de l'Aménagement du territoire (M. Rocard) définit le périmètre de l'économie sociale en référence aux statuts: "Elle a pour mission d'aider au développement des mutuelles, des coopératives, ainsi que des associations dont les activités de production les assimilent à ces organismes, qui interviennent dans le domaine de l'économie sociale. "

Dix ans plus tard, le décret du 28 octobre 1991 crée une Délégation générale à l'innovation sociale et à l'économie sociale (toujours Dies); celle-ci est rattachée au ministre des Affaires sociales et de l'Intégration (Jean-Louis Bianco). Elle a "pour mission de proposer et de coordonner les mesures destinées à favoriser le développement des coopératives, des mutuelles et des associations intervenant dans le secteur de l'économie sociale; de promouvoir en ce domaine les initiatives et les innovations; de coordonner les études et les projets tendant à l'harmonisation des dispositions qui sont applicables aux organismes mentionnés ci-dessus et aux organismes s'assignant un but similaire ".

Le périmètre est élargi, le lien entre l'ES et l'innovation est créé, mais le rattachement au ministère des Affaires sociales marque le début d'une lente évolution vers une conception de l'ESS limitée à la sphère sociale, amputée de sa dimension économique.

\section{... aux structures innovantes}

Le décret du 5 octobre 1995 institue une Délégation interministérielle à l'innovation sociale et à l'économie sociale (Dieses). Certes, de générale, la délégation est affirmée "interministérielle ", ce qu'elle était de facto en 1981, mais elle est désormais placée auprès du ministre chargé de l'Intégration et de la Lutte contre l'exclusion (Premier ministre: A. Juppé).

Dans le décret du 27 mai 1998 portant extension des missions de la Délégation interministérielle à l'innovation sociale et à l'économie sociale, la Dieses est rattachée au ministre de l'Emploi et de la Solidarité (Martine Aubry). La délégation couvre désormais toutes les associations et non pas uniquement les associations employeurs.

Puis, par décret du 7 avril 2000 relatif aux attributions déléguées au secrétaire d'Etat à l'Economie solidaire, Guy Hascoët, "par délégation de la ministre de l'Emploi et de la Solidarité, est en charge de la promotion et du développement de l'ESS. A ce titre, il prépare et propose notamment 
toutes les mesures intéressant la vie associative, la coopération, la mutualité, les nouvelles formes d'activité et de partenariat, ainsi que l'innovation économique et sociale...; il dispose de la Dieses et en tant que de besoin de la $D G E F P$ (Direction générale del'emploi et de la formation professionnelle), de la $D A G P B$ (Direction de l'administration générale du personnel et du budget), de l'Igas (Inspection générale de l'action sociale)».

\section{Une menace de disparition au profit de la seule innovation sociale}

Dans une allocution devant le Conseil national de lutte contre la pauvreté et l'exclusion sociale, le 16 septembre 2005, Dominique de Villepin, Premier ministre, déclare: "Mais inventer, rester à la pointe de l'innovation sociale, cela suppose d'être en dehors des contraintes de gestion qui sont celles des administrations locales et centrales. C'est pourquoi j'ai décidé de transformer la Délégation interministérielle à l'innovation sociale et à l'économie solidaire en Délégation interministérielle à l'innovation et à l'expérimentation sociale (l'économie sociale n'est pas nommée). Elle sera directement rattachée à Jean-Louis Borloo et Catherine Vautrin. Les missions actuelles de la Diieses en ce qui concerne la vie associative resteront auprès de JeanFrançois Lamour. " La nouvelle délégation sera une structure légère; elle aura pour mission de rassembler les pratiques les plus innovantes et efficaces en France, mais aussi à l'étranger, et de définir les conditions de leur application. La délégation devra également accompagner les grands chantiers décidés par le gouvernement, qu’il s'agisse de la réforme des minima sociaux (RSA), de la mise en place de chartes territoriales de cohésion sociale ou bien encore du développement du microcrédit. Elle travaillera en étroite relation avec le Conseil national de lutte contre les exclusions. "Je propose également la création, dès 2006, d'un fonds départemental d'innovation et d'expérimentation sociale doté de 20 millions d'euros, ajoute le Premier ministre. Géré par les préfets et les Ddass (directions départementales des affaires sanitaires et sociales), il permettra de soutenir les innovations locales et de favoriser, par le biais d'appels à projets, aussi bien le développement du microcrédit, que la création du guichet unique d'accueil, la mise en réseau d'acteurs sur un territoire ou bien de soutenir des démarches d'évaluation [...]."

Cette intervention et ses conséquences pour la Diieses ne peuvent s'entendre que par rapport au contexte (forte augmentation du taux de chômage, des exclusions et de la précarisation) et parmi ces éléments de contexte, il convient de citer le rapport de la commission "Familles, vulnérabilité, pauvreté ", présidée par Martin Hirsch: "Au possible, nous sommes tenus."

L'économie sociale n'est même pas nommée; la Diies est stigmatisée. Le Ceges, Conseil des entreprises, employeurs et groupements de l'économie sociale, monte au créneau; le terme " économie sociale » est ajouté sous la pression des acteurs. La Diieses apparaît comme un outil à disposition de Martin Hirsch, qui lance le RSA avec une importante enveloppe budgétaire abondée en partie par le budget de la Diies. 
L'ES au service de l'intérêt général et des politiques publiques (IAE)... Dans le décret du 13 février 2006, une Délégation interministérielle à l'innovation, à l'expérimentation sociale et à l'économie sociale est créée (Diieses), rattachée au ministre chargé de l'Emploi, de la Cohésion sociale et du Logement (Jean-Louis Borloo). Elle est chargée de "soutenir et promouvoir, au niveau national, le développement des activités d'intérêt général innovantes pour la mise en auvre des politiques publiques, en liaison notamment avec les collectivités publiques et les représentants des secteurs associatifs, coopératifs et mutualistes, d'identifier les initiatives prises à cet égard en France et à l'étranger, et de procéder à leur évaluation; à ce titre, elle participe aux travaux du Conseil national des politiques de lutte contre la pauvreté et l'exclusion sociale et du Conseil national de l'insertion par l'activité économique».

Le deuxième paragraphe reprend ce qui a été écrit précédemment concernant les mesures destinées à favoriser le développement des coopératives, des mutuelles et des associations. Elle se voit ainsi fixer pour missions "d'assurer une concertation entre les pouvoirs publics et les organismes intervenant dans le domaine de l'ES [et] de répartir les crédits du Fies(Fonds interministériel d'expérimentation sociale). La Diieses dispose de correspondants locaux"

\section{... avant d'être fondue dans l'action sociale en $\mathbf{2 0 1 0}$}

Dans le décret du 25 janvier 2010 relatif à l'administration centrale des ministères chargés des Affaires sociales et portant création d'une Direction générale de la cohésion sociale, il est précisé que "la DGCS est chargée de la conception, du pilotage et de l'évaluation des politiques publiques de solidarité, de développement social et de promotion de l'égalité favorisant la cohésion sociale. Elle veille à la cohérence nationale et territoriale de ces politiques [...]. Elle soutient et promeut le développement de l'ESS, ainsi que les innovations qui y concourent [...]. Elle est chargée d'animer et de coordonner l'action des pouvoirs publics et des ministères concernés en matière de politique d'innovation, d'expérimentation sociale et d'ESS [...]. Le directeur général de la cohésion sociale est délégué interministériel à la famille, délégué interministériel aux droits des femmes et à l'égalité entre les hommes et les femmes et délégué interministériel à l'innovation, à l'expérimentation sociale et à l'économie sociale ».

Le remaniement ministériel du 15 novembre 2010 a confirmé, quant à lui, la séparation entre les différentes catégories d'associations, puisque la jeunesse et la vie associative sont rattachées au ministre de l'Education nationale, Luc Chatel, alors que l'ESS est rattachée à Roselyne Bachelot, ministre des Solidarités et de la Cohésion sociale. C'est en 2004 que Jean-François Lamour, ministre des Sports, est devenu ministre des Sports, de la Jeunesse et de la Vie associative, invalidant la circulaire de 1998 du Premier ministre Lionel Jospin sur le développement de la vie associative, qui confiait alors à la Diieses cette compétence pour l'ensemble du champ associatif. 
Le récent gouvernement Ayrault ne semble pas avoir rompu avec cette séparation entre une vie associative rattachée au ministère du Sport et de la Jeunesse et une économie sociale et solidaire aujourd'hui directement intégrée à Bercy, semblant ainsi opposer la promotion de la citoyenneté et le soutien à l'activité économique et à l'emploi.

\section{1-2010 : les vicissitudes de la délégation}

Ces vicissitudes de la Délégation à l'économie sociale (Dies, Diies, Diieses...; voir annexe) sont dues à une succession d'évolutions, d'événements de nature et d'importance diverses, qui ont pesé et entraîné sa progressive marginalisation et son affaiblissement au sein de l'administration.

En vingt-neuf ans, la délégation a ainsi connu:

- onze délégués (ce qui fait une moyenne de 2,6 ans par délégué), dont les profils sont très divers (haute administration, milieu politique...); seul le premier délégué, Pierre Roussel, était issu du sérail (MGEN);

- un déménagement tous les vingt-quatre mois en moyenne;

- des personnels peu nombreux au sein de la structure Dies, auxquels furent adjoints quelques « cas sociaux » de par des volontés supérieures ou des inadvertances de l'administration;

- l'émergence relativement récente des agences fonctionnant sur le mode privé (Agence des services à la personne, ANSP; Agence pour la cohésion sociale, ACSE; Agence nationale pour l'autonomie des personnes âgées, ANSA...), désignant les délégations comme " ringardes ";

- la disparition (retraites, déplacements, non-prise en compte du sujet par les hiérarchies et les cabinets, etc.) du réseau de fonctionnaires compétents en matière d'ESS et de vie associative, au sein de l'administration centrale (mais aussi décentralisée), qui assurait une véritable transversalité et interministérialité, à tel point que le rapport de Francis Vercamer a bien souligné la nécessité d'en reconstruire un autre;

- certains effets de la révision générale des politiques publiques (RGPP). Les décrets et leur libellé ne sont évidemment pas anodins. Ils reflètent, au niveau des pouvoirs publics, un manque de culture sur le sujet, voire l'appréciation souvent partielle et partiale de l'ES ou de l'ESS par les décideurs publics, qui dès lors en déterminent ex abrupto et en partie l'objet (d'où ses nombreuses fluctuations au cours du temps).

Retenons la date de 1991 comme étape importante dans une conception qui au départ fut économique et qui désormais est sociale. Sans omettre les opportunités stratégiques et les aspects politiciens, les « batailles de territoires " entre les départements ministériels et les egos des ministres..., une lacune récurrente est évidente: la non-prise en compte globale, transversale et véritablement interministérielle de l'ESS par les pouvoirs publics, qui persiste depuis trente ans. Finalement, il est peut-être plus simple pour les décideurs publics et privés de considérer que les banques et les mutuelles de l'ES font partie intégrante du secteur bancaire et assurantiel au sein de l'économie générale du pays et que le reste de l'ES 
(8) "Faire mouvement " était le thème des lres assises du Ceges, le jer octobre 2008 (voir "Actualités ", Recma, $\left.n^{\circ} 310\right)$. Les Iles, le 16octobre2009, ontétéconsacrées à l'entrepreneuriat social ("En bref", Recma, $\left.n^{\circ} 314\right)--$ [NDLR]. (associations, fondations, insertion, entreprises émergentes, entreprises sociales), à haute fonction réparatrice et innovatrice, est bien utile socialement et constitue la seule ESS.

\section{Un défaut de projet commun de la part de l'ESS}

Dans ce contexte général, sommairement rappelé ici, il ne serait pas juste de ne pas évoquer une société civile qui peine à se penser organisée pour imposer ses points de vue et une faiblesse des managers de l'ESS à s'entendre pour faire un projet politique digne de ce nom et à y consacrer des moyens ${ }^{(8)}$.

Cela ne renvoie-t-il pas à une certaine image que donne d'elle-même l'ESS? Ses responsables de secteur n'agissent-ils pas trop souvent indépendamment les uns des autres, loin d'une indispensable unité d'action et d'une intercoopération cohérente avec leurs valeurs?

La capacité pédagogique, politique et de lobbying de certains des acteurs et des responsables de l'ES ou de l'ESS (pas toujours les mêmes selon les époques) a certainement "imprégné » l'orientation générale des politiques publiques relatives au secteur. Mais étaient-elles destinées à une famille du secteur seulement ou visaient-elles l'intérêt général de l'ensemble de l'ESS?

\section{De la coproduction à la mise en concurrence}

Ces hésitations sur le rôle et la nature de l'ESS, entre apport à la démocratie économique et apport à la solidarité sociale, entre formes établies et formes émergentes, entre approches sectorielles et approches transversales, s'inscrivent néanmoins dans des évolutions générales convergentes des politiques publiques. Malgré un discours croissant sur la coproduction des politiques publiques, les relations entre l'ESS et les pouvoirs publics n'échappent pas au processus de dérégulation. Si les années 60-70 étaient marquées par une certaine cogestion des politiques avec les grandes fédérations nationales, à partir des années 80 l'approche partenariale est percutée par une promotion croissante de l'efficacité de la concurrence pour augmenter l'offre de services et faire baisser les prix.

\section{La phase de la cogestion}

A partir de l'après-guerre, dans plusieurs secteurs, notamment social, sanitaire et social, médico-social, les acteurs mutualistes et associatifs se sont impliqués dans une cogestion avec les pouvoirs publics. Ces domaines d'intérêt général nécessitaient des politiques publiques pour tous: la santé, la retraite, la protection sociale, le chômage. L'expérience des retraites ouvrières et paysannes (ROP), par exemple, bien qu'elle se soldât par un échec, ne fut pas inutile, car des leçons en furent tirées ultérieurement, concernant la place des mutuelles dans l'organisation 
(9) Devenu L'Economie sociale partenaire de l'école de la République (L'Esper) en décembre 2010 (NDLR). du système de protection sociale. Ainsi, leur rôle en tant que cogestionnaire de l'Assurance maladie a été établi par l'ordonnance du 19 octobre 1945 et constamment réaffirmé jusqu’au Code de la mutualité de 2001.

C'est aussi la loi du 30 juin 1975, dite des CEuvres, qui fonde et organise le secteur social et médico-social, associant de grands réseaux associatifs réunis dans l'Union nationale interfédérale des œuvres et organismes privés sanitaires et sociaux (Uniopss), jusqu'à la loi du 2 janvier 2002 rénovant l'action sociale et médico-sociale.

De même dans le sport, le Comité national olympique et sportif français (CNOSF) est tellement intégré aux politiques publiques que sa structuration épouse celle de l'administration.

Dans l'éducation, le Comité de coordination des œuvres mutualistes et coopératives de l'Education nationale (Ccomcen ${ }^{(9)}$ ) a longtemps réuni les acteurs de l'économie sociale éducative pour, d'une part, régler les questions de concurrence interne (notamment en termes d'assurance) et, d'autre part, former un interlocuteur du ministère de l'Education nationale.

Enfin, les coopératives ne sont pas en reste: la coopération agricole a été depuis ses origines un partenaire privilégié des politiques agricoles nationales, jusquaux différentes réformes de la Politique agricole commune (PAC).

\section{La phase du partenariat}

Cette cogestion, très sectorielle selon les découpages administratifs, est remise en cause à partir des années 80 , du fait de la décentralisation, d'une part, et des mutations des politiques publiques, d'autre part. Se dessine alors une approche dite plus «partenariale " (Draperi, 2007). Il s'agit de casser la "logique de guichet", les associations venant réclamer leur " dû » - la subvention annuelle qui les aide à fonctionner - aux administrations centrales ou décentralisées. Pour les financeurs, il s'agit de ne plus réfléchir en termes de "clientèle fidélisée et régulière ", mais de mieux évaluer les besoins et les demandes, en fonction des budgets disponibles, mais aussi de la nature et de la qualité des projets associatifs eux-mêmes. Il s'agit de créer d'autres relations basées sur une connaissance et une reconnaissance mutuelles, un dialogue " adulte " avec l'élaboration de la Charte des engagements réciproques entre l'Etat et les associations, en 2001, au moment de la commémoration du centenaire de la loi de 1901.

Il convient, parallèlement, de sécuriser les financements accordés. En matière de subventionnement, la volonté partagée du mouvement associatif et des pouvoirs publics est de développer les conventions pluriannuelles d'objectifs, qui instaurent des simplifications administratives, tel le dossier unique de demande de subvention, ou bien les bases du partenariat autour du projet associatif, avec l'obligation d'une évaluation, déterminée ensemble en amont et partagée (circulaires du $1^{\text {er }}$ décembre 
(10) Le quart réservataire a disparu en 2001, au bénéfice de l'article 54 accordant un droit de préférence " attribué, à égalité de prix ou à équivalence d'offres, à l'offre présentée par une Scop, par un groupement de producteurs agricoles, par un artisan, une société coopérative d'artisans ou par une société coopérative d'artistes ou par une entreprise adaptée ". La clause d'insertion est entrée dans le Code des marchés publics le 7 mars 2001 (moins de $2 \%$ des marchés en 2009). Sur toutes ces questions, et notamment la mise en concurrence des associations, voir www.patrickloquet.fr (NDLR).
2000, du 24 décembre 2002, du 16 janvier 2007 et du 18 janvier 2010). Ce sont les fruits des travaux de la Ire Conférence nationale de la vie associative, du premier semestre 2005 (trois rapports et quatre-vingtune propositions), en particulier ceux du groupe dédié aux questions de financement et de définition de la subvention, sur "la consolidation des relations contractuelles entre les pouvoirs publics et les associations ".

Des clarifications et des régulations pour une plus grande transparence et une meilleure efficacité sont mises en place sur la base de réflexions communes: services de l'Etat et représentants des divers ministères concernés, Conseil national de la vie associative au titre de ses nombreuses études très qualifiées, représentants des secteurs associatifs et de la Conférence permanente des coordinations associatives (CPCA). Ainsi naît l'instruction fiscale du 15 septembre 1998 - les circulaires relatives aux subventions ci-dessus mentionnées, la réforme du Code des marchés publics avec l'inclusion de clauses sociales et d'un mieux-disant social, d'un quart réservataire ${ }^{(10)}$. Par ailleurs, il est de plus en plus clair que des contrôles comme ceux exercés par les chambres régionales des comptes et la Cour des comptes ou par les inspections générales concernent et s'appliquent aux organisations et aux entreprises de l'ESS, tout comme des lois non spécifiques. Par exemple, la loi Sapin du 29 janvier 1993, relative à la prévention de la corruption et à la transparence de la vie économique et des procédures publiques, exige des associations plus de rigueur comptable et budgétaire, plus de contrôle et d'évaluation, mais aussi plus de concurrence.

\section{La phase de la mise en concurrence et de la loi du marché}

La mise en concurrence, en effet, s'est nettement accentuée. Elle est dénoncée par les associations et la CPCA, mais comment résister, faire à la fois respecter son identité spécifique et trouver des moyens pour concevoir et développer des projets et maintenir la structure et les emplois associatifs? Cette mise en concurrence actuelle est redoutable pour la démocratie et le projet associatif: elle se développe au moyen de la commande publique, des appels d'offres, des appels à projets, qui transforment les associations en concurrentes non seulement entre elles-mêmes, mais aussi avec d'autres entreprises individuelles ou lucratives. Cela les banalise et risque de modifier leur nature profonde, les faisant aller vers d'autres champs et déplaçant leurs frontières. Les associations deviennent "des opérateurs comme les autres " pour les donneurs d'ordre public, qui ne reconnaissent que leur activité (en termes de résultats immédiats et directs) et non leur mode de fonctionnement (en termes de processus et d'impact).

Plus insidieuse encore est l'instrumentalisation des associations et des autres acteurs du fait de leur acceptation de prendre en charge la gestion de dispositifs publics ou de missions dites de service public (Moreau, 2004). 


\section{Conclusion}

Les différents mouvements de l'ESS veulent-ils vraiment une politique publique qui les concerne tous et chacun, ensemble? Jusqu' ici, «l'esprit » qui a présidé aux travaux de la commission Vercamer a semblé répondre positivement à ces deux questions. Les mois qui viennent vont être les témoins de la réalité de cette volonté commune ou de son inexistence.

La loi-cadre sur l'ESS en préparation au Conseil supérieur de l'économie sociale et solidaire peut constituer une belle opportunité pour que cette volonté se concrétise. Il conviendrait donc que l'ESS reste mobilisée et soit très active, très inventive en matière de propositions à cet égard. C'est indispensable, mais cela ne suffira pas. Le temps est venu pour l'ESS de se pencher avec courage, détermination et célérité sur son avenir en tant qu'ESS. Celui-ci passera par une réelle intercoopération, une vivante transversalité. C'est déjà le cas sur certains territoires.

Et il n'y aura politique publique que si, d'un côté comme de l'autre, responsables de l'ESS (élus et salariés) et pouvoirs publics, est mise en place une condition suffisante et nécessaire: la transversalité. La Direction générale de la cohésion sociale, constituée de plusieurs cultures administratives, a participé à créer les conditions d'une transversalité active dont l'ESS a profité au sein du Conseil supérieur de l'économie sociale et solidaire.

Aujourd'hui, le rattachement du nouveau ministre délégué à l'ESS à Bercy répond aux attentes du mouvement coopératif, qui le salue comme "une reconnaissance de [son] poids socio-économique " (communiqué de Coop FR, 23 mai 2012), comme du Ceges. Espérons que le passage du rattachement "social " au rattachement "économique " prenne bien en compte la nature et l'apport socio-économiques de l'économie sociale et solidaire comme forme d'internalisation (et non de hiérarchisation) des dimensions économiques et sociales; qu'il ne se réduise pas à substituer à l'objectif de 1981, "soutenir l'ES pour accompagner l'économie publique dans la modernisation de la France " celui de "soutenir l'ESS pour accompagner l'économie privée dans le redressement productif de la France ». 


\section{Bibliographie}

Amblard C., 2001-2002, "Cent ans de pratique associative: un point de vue juridique ", Recma, $\mathrm{n}^{\text {os }} 282$ et 283.

Chaves R., 2011, «La loi espagnole d'économie sociale: évaluation du point de vue de la politique publique », Recma, $\mathrm{n}^{\circ} 321$.

Chomel A., 1994, " Note pour l'histoire de la Revue des études coopératives (1921-1994)", Recma, $\mathrm{n}^{\circ} 268$.

Draperi J.-F., 2007, Comprendre l'économie sociale: fondements et enjeux, Paris, Dunod, p. $90-120$.

Draperi J.-F., 2009, "Au bénéfice de la crise? Pour un projet d'économie sociale et solidaire ", Recma, $\mathrm{n}^{\circ} 313$.

Hiez D., 2005, «Les instruments de fonds propres des coopératives: vingt ans d'innovation législative ", Recma, $\mathrm{n}^{\circ} 295$.

Hipszman M., 2003, "Quand furent posées les fondations d'une politique de l'économie sociale ", Recma, n² 290.
Koulytchizky S., 2006, « Place de l'économie sociale dans la construction de l'intérêt général à partir de l'initiative locale, les "pays" en France ", Recma, n 302.

Laville J.-L. (dir.), 2007, L'économie solidaire, une perspective internationale, Paris, Hachette. Medef, 2002, "Concurrence: marché unique, acteurs pluriels, pour de nouvelles règles du jeu ", mai; http://archive.medef.com/medias/ upload/506_FICHIER.pdf.

Moreau J., 2002, « Pour une étude des rapports entre l'Etat et l'économie sociale: spontanéité et instrumentalisation", Recma, n ${ }^{\circ} 284$.

Pénin M., 2006, « Charles Gide est-il toujours d'actualité? ", Recma, nº 301.

Rocard M., 2001, "L'économie sociale et solidaire: une perspective Nord-Sud, discours de clôture ", Recma, n 282.

Soulage F., Hipszman M., 2004, "La loi du 20 juillet 1983, première loi d'économie sociale ", Recma, no 291. 


\section{Annexe Intitulés de la délégation à travers les décrets}

- 1981 à 1991 : Délégation à l'économie sociale.

- 1991 à 1995: Délégation générale à l'innovation sociale et à l'économie sociale.

- 1995 à 2006: Délégation interministérielle à l'innovation sociale et à l'économie sociale.

- 2006 à 2010: Délégation interministérielle à l'innovation, à l'expérimentation sociale et à l'économie sociale.

- Décret n ${ }^{\circ}$ 81-1125 du 15 décembre 1981 (J0 du 20 décembre 1981) portant création d'une Délégation à l'économie sociale auprès du Premier ministre et mise à la disposition du ministre d'Etat, ministre du Plan et de l'Aménagement du territoire (on fait référence à la Dies en relation avec son caractère interministériel, qui n'est cependant pas inclus dans l'intitulé de la structure créée).

- Décret n n $^{91-1133 ~ d u ~} 28$ octobre 1991 (J0 du 31 octobre 1991) portant création d'une Délégation générale à l'innovation sociale et à l'économie sociale auprès du ministre des Affaires sociales et de l'Intégration (DGISES, mais on continue à faire référence à la Dies et à son caractère interministériel).

- Décret $n^{\circ}$ 95-1083 du 5 octobre 1995 (J0 du 8 octobre 1995) instituant une Délégation interministérielle à l'innovation sociale et à l'économie sociale auprès du ministre chargé de l'Intégration et de la Lutte contre les exclusions - décret modifiant celui de 1991 - (on fait référence à la Diises).

- Décret n ${ }^{\circ}$ 98-410 du 27 mai 1998 (J0 du 29 mai 1998) portant extension des missions de la Délégation interministérielle à l'innovation sociale et à l'économie sociale auprès du ministre chargé de l'Emploi et de la Solidarité (on fait référence à la Diises).

- Décret n ${ }^{\circ}$ 2000-308 du 7 avril 2000 (J0 du 8 avril 2000) relatif aux attributions déléguées au secrétaire d'Etat à l'Economie solidaire (par délégation du ministre de l'Emploi et de la Solidarité, le secrétaire d'Etat à l'Economie solidaire chargé de la promotion et du développement de l'économie sociale et solidaire).

- Décret n $\mathbf{n}^{\circ}$ 2000-685 du 21 juillet 2000 relatif à l'organisation de l'administration centrale du ministère de l'Emploi et de la Solidarité et aux attributions de certains services (article 10).

- Décret $n^{\circ}$ 2006- 151 du 13 février 2006 (J0 du 14 février 2006) instituant une Délégation interministérielle à l'innovation, à l'expérimentation sociale et à l'économie sociale auprès du ministre chargé de l'Emploi et de la Cohésion sociale. La Délégation interministérielle à l'innovation sociale et à l'économie sociale devient la Délégation interministérielle à l'innovation, à l'expérimentation sociale et à l'économie sociale (on fait référence désormais à la Diieses).

- Décret n $\mathrm{n}^{\circ}$ 2010-95 du 25 janvier 2010 (J0 du 26 janvier 2010) relatif à l'administration centrale des ministères chargés des Affaires sociales et portant création d'une Direction générale à la cohésion sociale (DGCS) et suppression des diverses délégations dont la Diieses. 